\title{
Research Paper \\ The Effect of Locally Produced Unstable Shoes on Foot Plantar Pressure During Walking Among Healthy Male Students
}

\author{
Siavash Etemadi Nejad ${ }^{1}\left(\mathbb{0},{ }^{*}\right.$ Saed Ahmadi Ganjeh ${ }^{1}\left(\mathbb{C}\right.$, Jamshid Yazdani Charati², Esmail Hoseini Nejad ${ }^{3}$
}

\author{
1. Department of Occupational Health Engineering, School of Health, Mazandaran University of Medical Sciences, Sari, Iran \\ 2. Department of Epidemiology \& Biostatistics, School of Health, Mazandaran University of Medical Sciences, Sari, Iran. \\ 3. Department of Sports Biomechanics, Faculty of Physical Education and Sports Sciences, University of Mazandaran, Babolsar, Iran
}

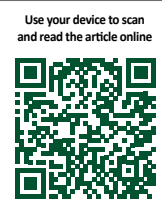

Cittation: Etemadi Nejad S, Ahmadi Ganjeh S, Yazdani Charati J, Hoseini Nejad E. [The Effect of Unstable Shoe on Shoe Sole Pressure During Walking Among Healthy Male Students (Persian)]. Journal of Sport Biomechanics. 2018; 4(3):52-61. https://doi. org/10.32598/biomechanics.4.3.52

dol' https://doi.org/10.32598/biomechanics.4.3.52

Keywords:

Walking, Foot biomechanics, Unstable shoes

\begin{abstract}
Objective: Today, various shoes have been developed to reduce injury and improve performance during walking. The aim of this study was to evaluate the effect of locally produced unstable shoes on foot plantar pressure during walking in healthy male students.

Methods: Participants were 20 healthy male students (Mean $\pm S D$ of height $=178.96 \pm 3.92 \mathrm{~cm}$, Mean $\pm S D$ of age $=27 \pm 3$ years, Mean $\pm S D$ of mass $=73.99 \pm 6.6 \mathrm{~kg}$ and $\mathrm{EU}$ shoe size $=42$ ). They were evaluated under four wearing conditions: Locally produced unstable shoes, unstable shoes produced abroad, regular control shoes, and barefoot. The maximum foot plantar pressure was measured in ten plantar areas by a footscan system while walking. The generalized linear model and repeated measures Analysis of Variance (ANOVA) and/or Friedman test at a significance level of $0.05(\mathrm{P}<0.05)$ were used for analyzing data using SPSS V. 23 software.

Results: Maximum foot plantar pressure during walking under four different conditions was significantly different only in three plantar regions of hallux, lesser digits, and metatarsal $5(\mathrm{P}<0.05)$.

Conclusion: Suggest the inefficiency of unstable shoes manufactured in Iran in adjusting the plantar pressure in individuals.
\end{abstract}

\section{Extended Abstract}

\section{Introduction}

W

alking is a perfectly coordinated and complex activity performed in collaboration with the musculoskeletal and nervous systems. The mechanical behavior of these organs has been well identified and analyzed. Such data have been gained by a better understanding of motor organs and relying on sensitive measurement equipment
[1]. Various methods have been used to reduce foot pressure during gait, including wearing shoes, insole, and socks, as well as using surgical procedures and injections [2]. Foot orthoses compensate foot pressure to some extent; however, the biomechanical impact of such tools is unclear [3].

Although prior research has investigated unstable shoe effects, the productivity of such footwear remains unaddressed. Furthermore, unstable shows produced locally are widely available in shoe stores; however, their efficacy and biological impact, compared to those made by foreign

\section{* Corresponding Author:}

Saed Ahmadi Ganjeh, Msc.

Address: Department of Occupational Health Engineering, School of Health, Mazandaran University of Medical Sciences, Sari, Iran.

Tel: +98 (917) 1450095

E-mail: saedahmadi1364@gmail.com 
countries, have been overlooked. There is no consensus on the biomechanical effects of these shoes on the body. This study examined the effect of locally produced unstable shoes on foot plantar pressure. To this aim, the maximum pressure in ten plantar regions (hallux, lesser digits, 1-5 metatarsals, midfoot, medial, and lateral heels) were considered between different conditions in terms of foot coverage while walking. We aimed to explore the effect of locally produced shoes on foot plantar pressure variables during walking, compared to those produced abroad, conventional shoes, and bare feet condition in healthy people.

\section{Participants and Methods}

In total, 20 subjects were selected among healthy rightfooted male students. For evaluating the plantar arch, the navicular drop was measured using the common method [10]. Accordingly, the difference in navicular height between standing and sitting positions were measured and a range of 5-9 mm was considered as normal [10]. To measure the knee varus, the subject stood with lower body naked, knees in full extension, ankles stuck together, and the patella toward the anterior direction. Then, the distance between the two inner epicondylitis of the knee was measured; values $<2 \mathrm{~cm}$ were considered as natural [9]. To measure the knee valgus, the Tibial Mechanical Anatomical (TMA) angles of $6-9^{\circ}$ perpendicular to the axis were considered normal [11]. To avoid immediate effects, before applying walking conditions with different shoes, the study subjects randomly wore the shoes for 20 minutes and began walking on the lab ground.

The walking route was considered about $15 \mathrm{~m}$ and the subjects started walking from an 8-m distance from the foot plantar pressure gauge for 3 attempts. The average value of these three attempts was recorded as the subject's rate on walking test under all 4 footwear conditions (locally produced unstable shoes, unstable shoes produced abroad, normal control shoes, and bare feet). The study subjects randomly performed walking test protocols; then, the obtained data were measured and normalized for each subject. A foot scan system (RS-scan, Belgium) with a sampling frequency of $250 \mathrm{~Hz}$ and a size of $1 \times 0.4 \mathrm{~m}$ was used for measuring variables related to foot plantar pressure.

\section{Results}

Variables related to foot plantar pressure, including the surface area of the regions as well as forces applied to it, were combined and analyzed (Table 1).

\section{Discussion}

The obtained results suggested that the maximum foot plantar pressure on the regions of 1-4 metatarsals, midfoot,

Table 1. Mean \pm SD values of maximum pressure applied to 10 plantar regions under 4 different conditions during gait

\begin{tabular}{|c|c|c|c|c|}
\hline \multirow{3}{*}{ Variables } & \multicolumn{4}{|c|}{ Test Conditions } \\
\hline & \multicolumn{4}{|c|}{ Mean \pm SD } \\
\hline & Bare Feet & Normal Shoes & $\begin{array}{c}\text { Unstable Shoes Pro- } \\
\text { duced Abroad }\end{array}$ & $\begin{array}{l}\text { Unstable Shoes Pro- } \\
\text { duced Locally }\end{array}$ \\
\hline Maximum pressure on hallux & $6.074 \pm 3.379$ & $5.789 \pm 4.913$ & $5.074 \pm 2.880^{\star \star}$ & $0.357 \pm 0.022^{\star *}$ \\
\hline Maximum pressure on lesser digits & $5.398 \pm 5.484^{*}$ & $6.368 \pm 4.201$ & $7.281 \pm 0.305$ & $11.661 \pm 9.642^{*}$ \\
\hline Maximum pressure on metatarsal 1 & $6.638 \pm 1.019$ & $6.119 \pm 1.531$ & $5.843 \pm 1.673$ & $6.528 \pm 3.834$ \\
\hline Maximum pressure on metatarsal 2 & $10.793 \pm 9.622$ & $8.619 \pm 6.931$ & $6.735 \pm 1.976$ & $7.622 \pm 2.458$ \\
\hline Maximum pressure on metatarsal 3 & $10.531 \pm 6.345$ & $8.660 \pm 5.079$ & $7.375 \pm 2.341$ & $8.898 \pm 3.338$ \\
\hline Maximum pressure on metatarsal 4 & $8.420 \pm 4.087$ & $7.507 \pm 2.297$ & $6.517 \pm 3.349$ & $20.663 \pm 7.530$ \\
\hline Maximum pressure on metatarsal 5 & $5.550 \pm 1.991^{*}$ & $6.678 \pm 1.299$ & $6.952 \pm 1.827$ & $8.175 \pm 2.376^{\star}$ \\
\hline Maximum pressure on midfoot & $4.225 \pm 1.878$ & $4.384 \pm 2.292$ & $5.007 \pm 0.797$ & $5.208 \pm 1.701$ \\
\hline Maximum pressure on the medial heel & $6.969 \pm 2.600$ & $5.492 \pm 2.398$ & $5.621 \pm 2.002$ & $6.441 \pm 3.666$ \\
\hline Maximum pressure on the lateral heel & $6.126 \pm 1.618$ & $5.722 \pm 2.200$ & $5.065 \pm 2.288$ & $5.183 \pm 2.515$ \\
\hline
\end{tabular}

* Significant difference between two conditions of walking with unstable shoes produced locally and with bare feet $(\mathrm{P}<0.05)$.

** Significant difference between two conditions of walking with unstable shoes produced locally and abroad $(\mathrm{P}<0.05)$. 
medial, and lateral heels did not depend on the footwear type during gait. Maximum foot plantar pressure on hallux while walking with the foreign-made unstable shoes significantly reduced, compared to the locally-produced ones. There was no significant difference between other investigated conditions.

Maximum foot plantar pressure on lesser digits while walking with the locally-made unstable shoes significantly increased, compared to bare feet condition; however, there was no significant difference between other explored conditions. Maximum foot plantar pressure on metatarsal 5 while walking with the locally-made unstable shoes also significantly increased, compared to walking with bare feet. However, no significant difference was detected between other study conditions.

There were significant changes under different footwear conditions in hallux, lesser digits, and metatarsal 5 regions. The pressure difference in these plantar regions could belong to the rigidity and low adsorption properties of unstable shoes. These abnormal pressures could harm the foot sole over time. Our achieved results are consistent with those of Stewart et al. [5] but against those of Kavros et al. [14, 15].

\section{Conclusion}

Locally-made unstable shoes significantly increased maximum pressure on lesser digits and metatarsal 5 regions, compared to bare feet condition as well as on hallux, compared to foreign-made unstable shoes. This finding indicates that these locally produced unstable shoes not only were unable to decrease the pressure on lesser digits and metatarsal 5 regions but also increased it. This highlights the inefficiency of unstable shoes manufactured in Iran in adjusting the plantar pressure in individuals.

\section{Ethical Considerations}

Compliance with ethical guidelines

A written informed consent was obtained from all participants and they were informed of the study objectives and methods.

Funding

This study was extracted from a thesis conducted in collaboration with the Department of Occupational Health and the Department of Ergonomics of the School of Public Health in Sari and the Health Assessment and Monitoring Center of the University of Mazandaran.

\section{Authors' contributions}

Conceptualization: Saed Ahmadi Ganjeh and Seyed Esmail Hoseini Nejad; investigation: Siavash Etemadi Nejad, Saed Ahmadi Ganjeh, and Jamshid Yazdani Charati; Review \& Editing: Saed Ahmadi Ganjeh

\section{Conflicts of interest}

The authors declare no conflict of interest.

\section{Acknowledgements}

The authors would like to thank the Health Assessment and Monitoring Center of the University of Mazandaran for their valuable cooperation. 


\title{
تأثير كفش نإِايدار بر فشار كف زيرين كفش هنكَام راهرفتن در دانشجويان يسر سالم
}

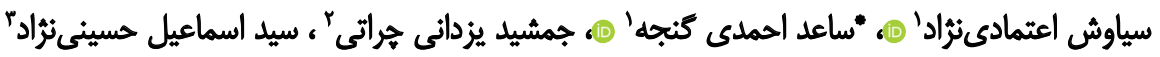

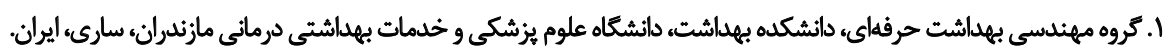

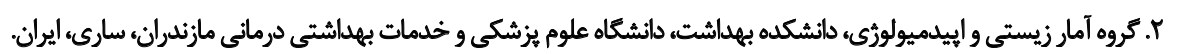 \\ ب. كروه بيومكائيك ورزشى و رفتار حركتى، دانشكده علوم ورزشي، دانشكاه مازئدران، بابلسر، ايراني.
}

\begin{abstract}
حكيد

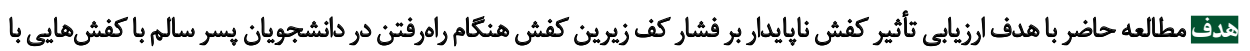

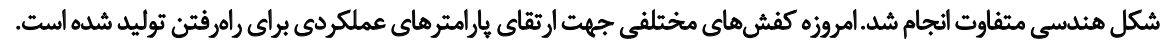

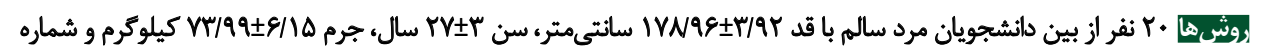

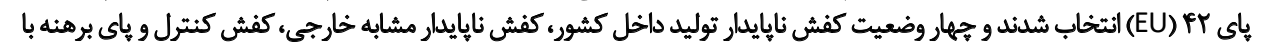

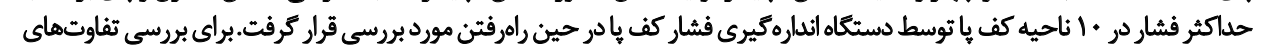

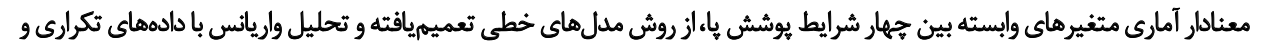

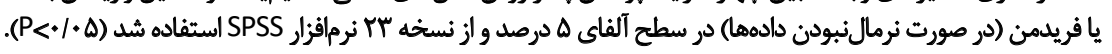

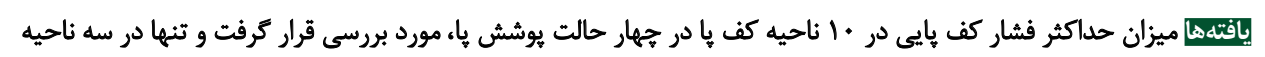

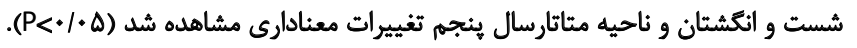
لتتيجه كيري نتايج به ناكارآمدى كفش نايايدار توليد داخل در تعديل فشار كف بائى در افراد اشاره دارد.
\end{abstract}

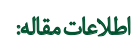

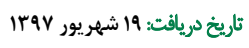

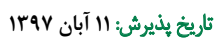

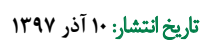

كليدوازهها:

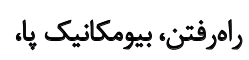
كفش نإيايدار

تأثير بيومكانيكى اين ابزارها هنوز بهطور كامل روشن نيست ["[].

datos

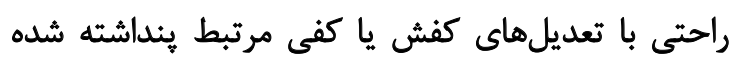

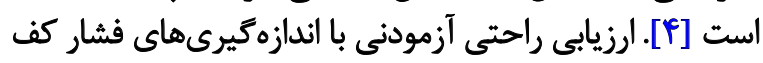

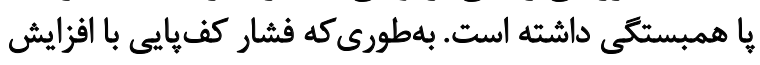

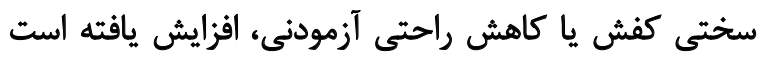

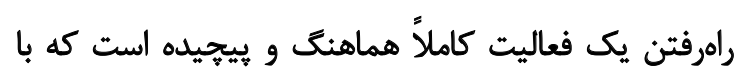

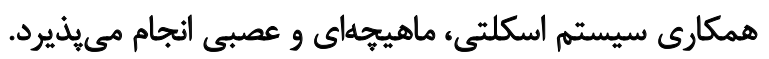

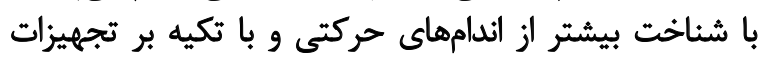

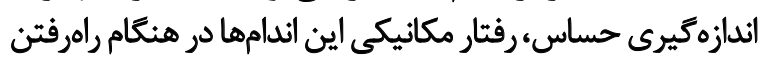

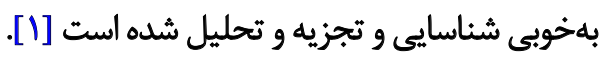

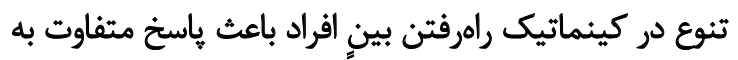

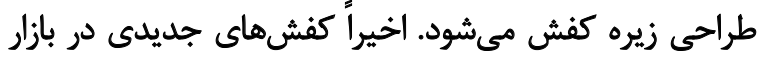

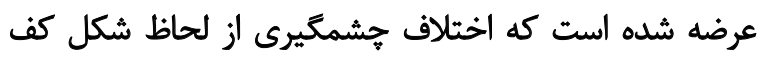

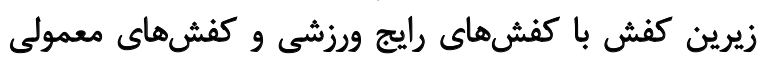

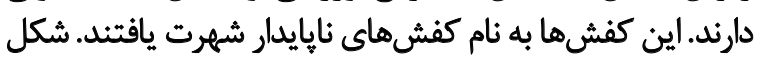

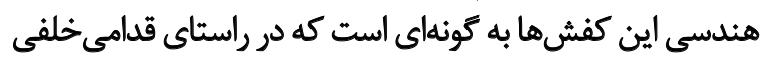
داراى انحنا بوده و متفاوت با ساير كفشه كائ است رايج است.

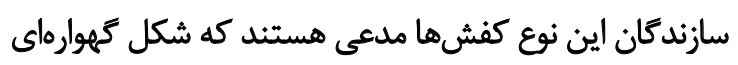

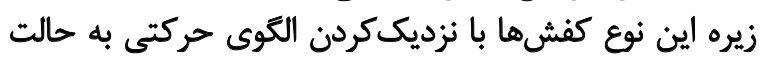

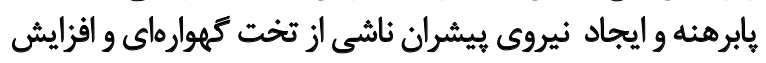

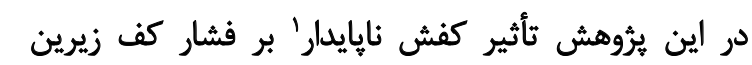

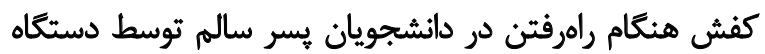

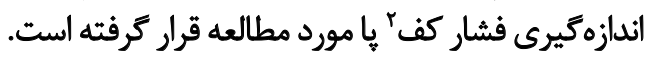

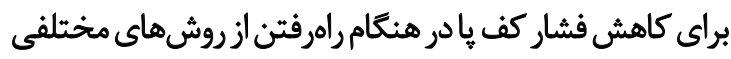

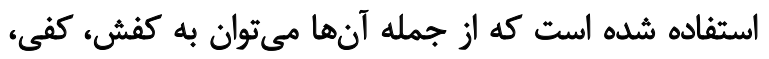

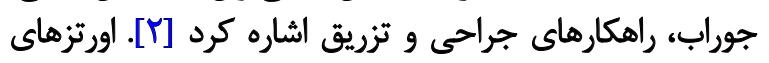

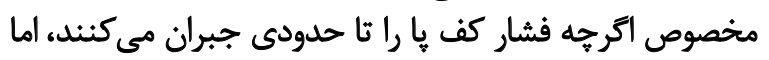

1. UnstableShoe

2. Foot Scane

*ون نويسنده مسئول:

ساعد احمدى كنجه نولين

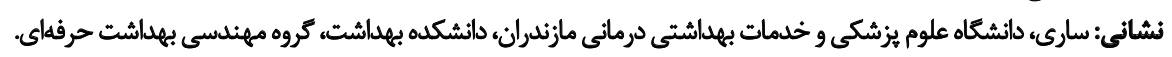

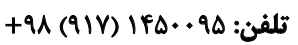
saedahmadi1364@gmail.com : بست الكترونيكى 
مرتبط به فشار در كف زيرين كفش افراد سالم تأثير دارد؟

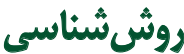

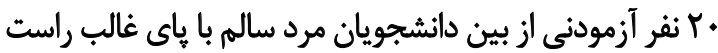

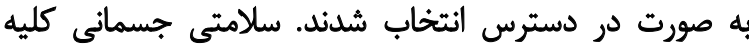

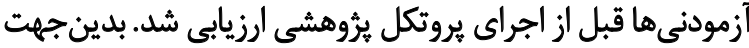

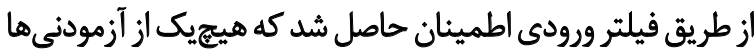

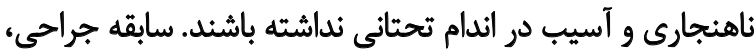

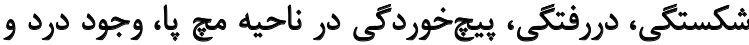

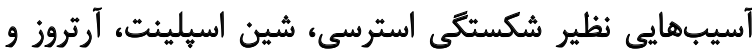

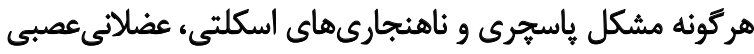

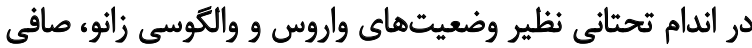

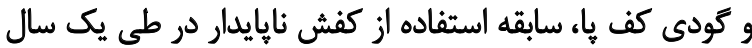

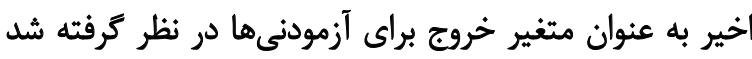

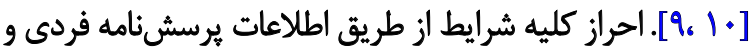

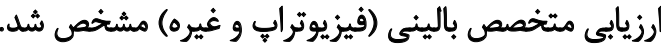
آزمودنىهاي يُروهش حاضر داراي مشخصات آنترويومتريكى،

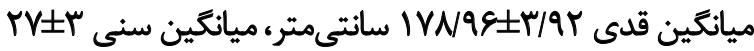

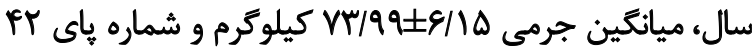

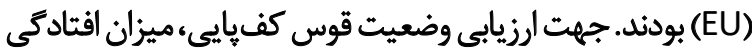

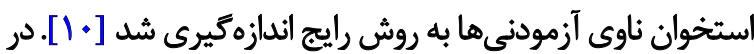

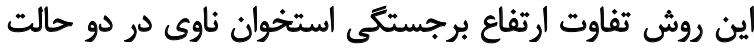

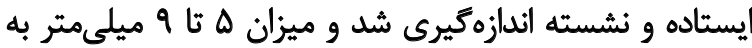

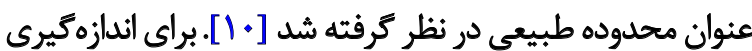

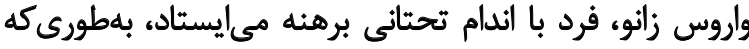

فعاليت عضلات كنترلى، باعث كاهش باركيرى مفصلى حين

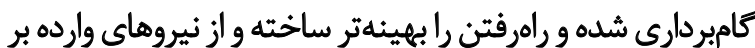

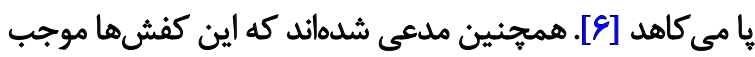

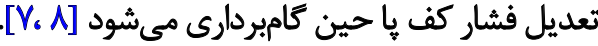
طراحى بهينه كف كفش و دانستن فشار وارد بر كف خارجى

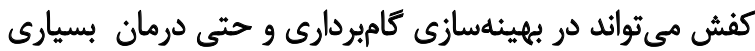

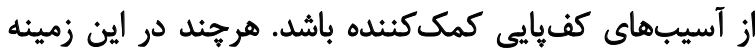

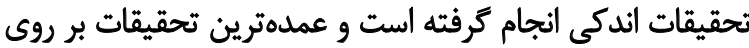
نيروهاى اعمالى به با بوده است.

علىرغم تحقيقات انجام شده در حوزه كفشهاى نايايدار هنوز

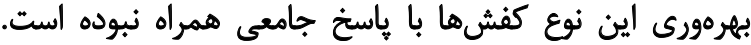

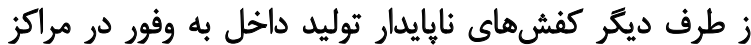

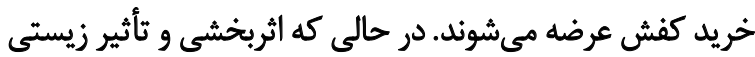

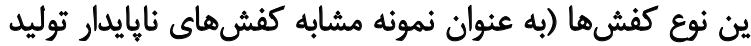

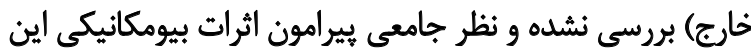
كفشها روى بدن وجود ندارد.

در اين يُروهش جهت ارزيابى فشار كف يا حداكثر فشار نقاط

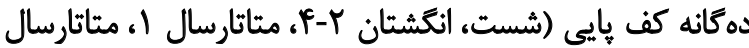

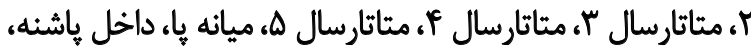

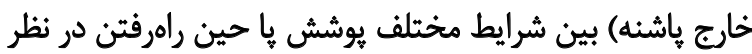
كرفته شلهه أست.

هدف از انجام اين بثروهش اين است كه آيا كفشهاي نائايدار

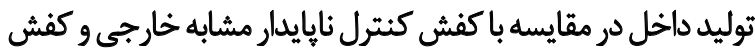

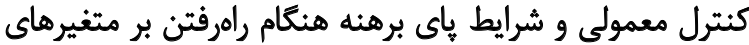

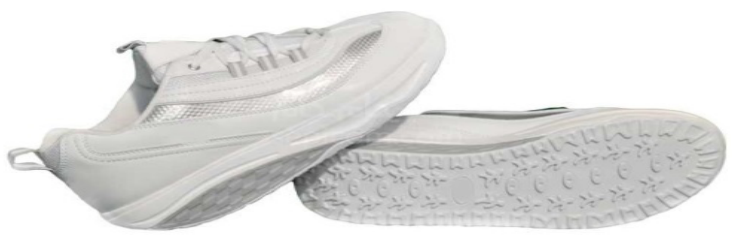

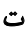

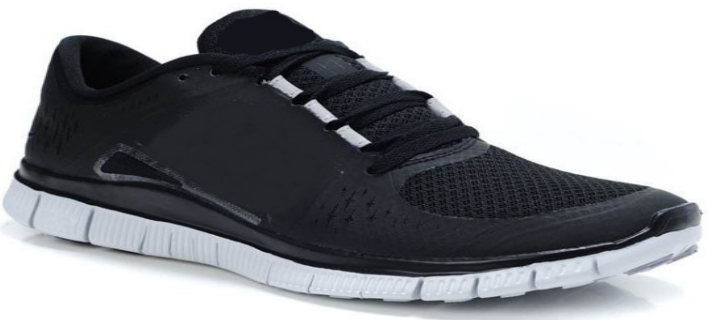

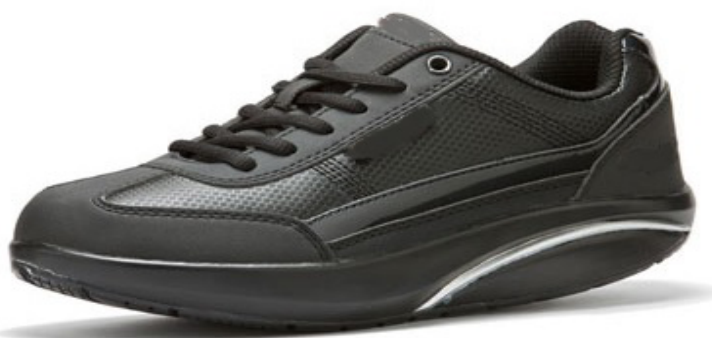


در كفش نايايدار مشابه خارجى در مقايسه با كفش نايايدار

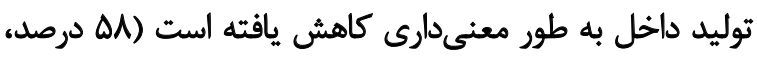

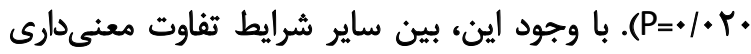

مشاهله نشد (P) (P)

حداكثر فشار ناحيه انكشتان بين سه شرايط يوشش يا تفاوت

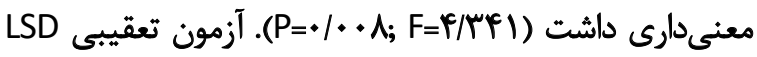

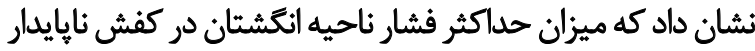

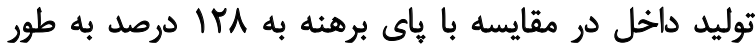

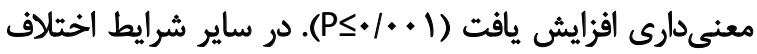

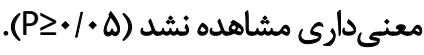

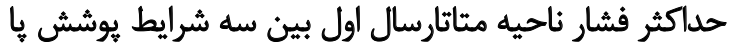

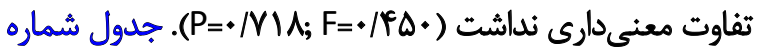

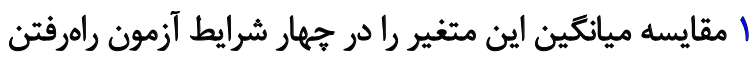

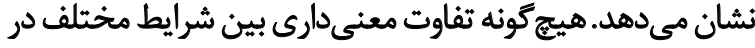

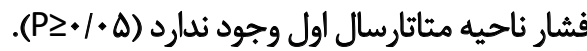

حداكثر فشار ناحيه متاتارسال دوم بين سه شرايط يوشش هاريا

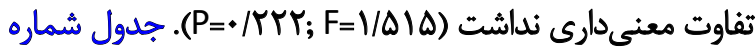

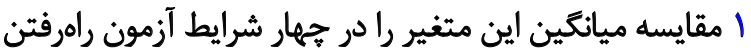

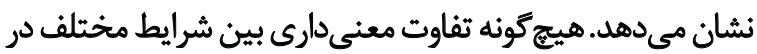

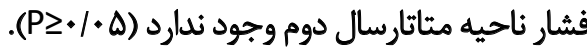

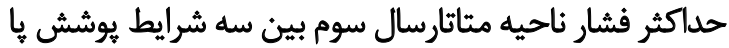

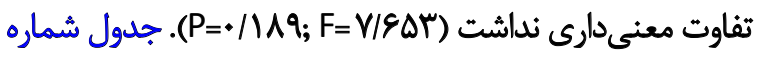

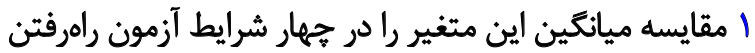

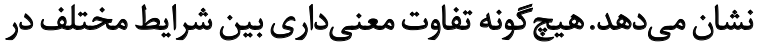

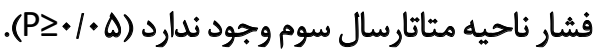

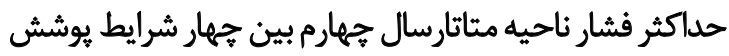

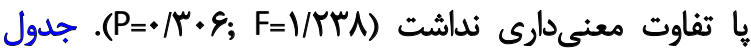

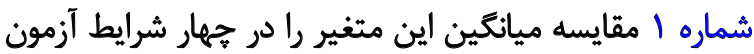

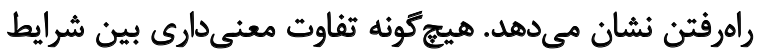

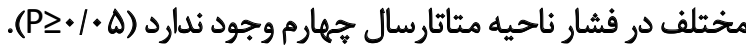

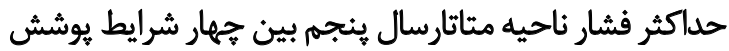

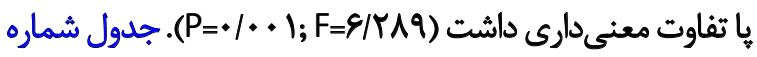

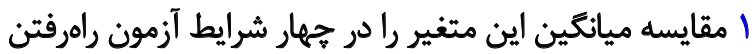

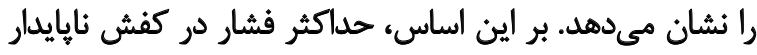

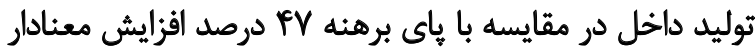

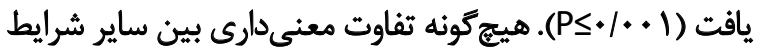

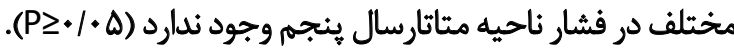

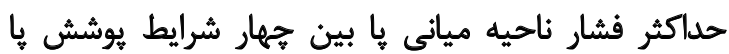

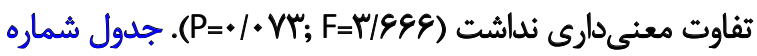

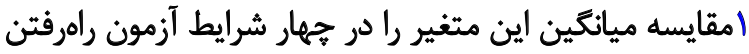

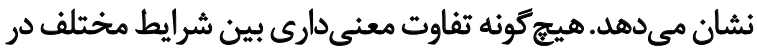

زانوها در باز شدن كامل"، قوزكها به هم جسبيده و كشكى

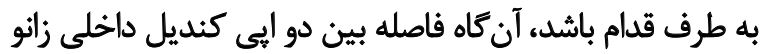

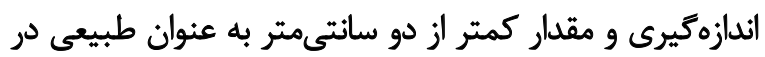

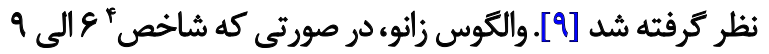

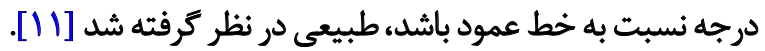

يروتكل راهرفتن: به منظور جلوكيرى از اثرات فورى، قبل از ازئ

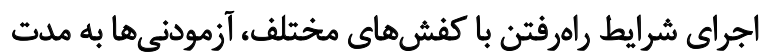

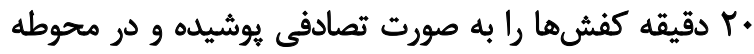

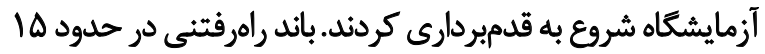

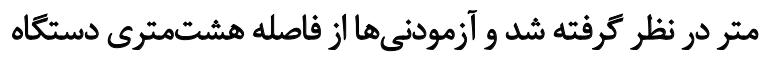

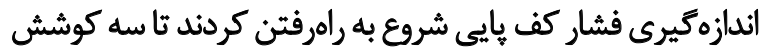

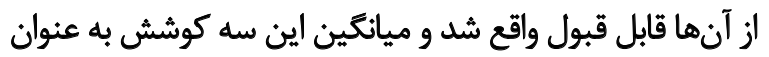

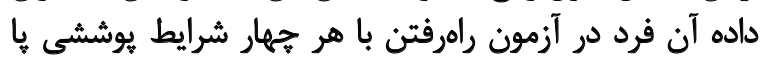

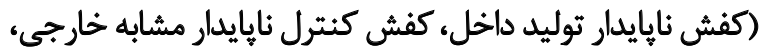

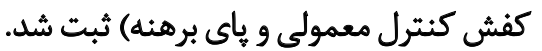

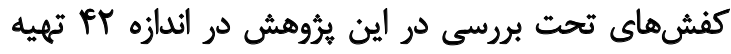

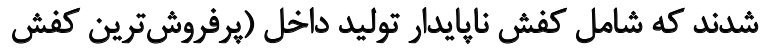

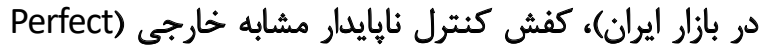

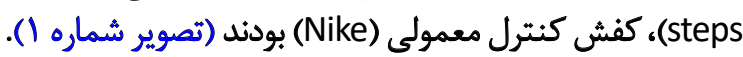

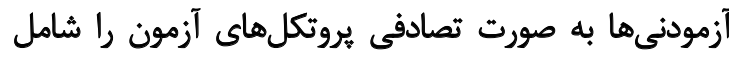

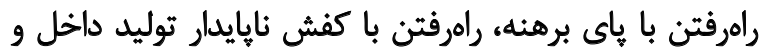

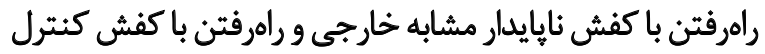

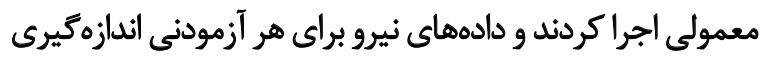

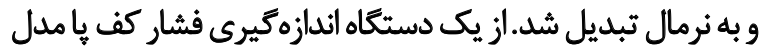

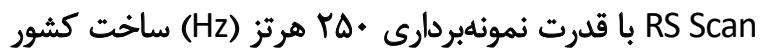

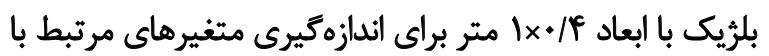

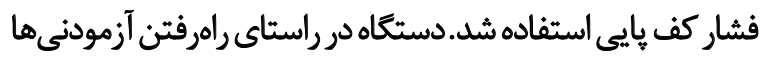

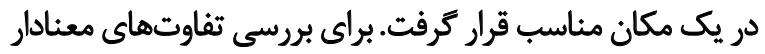

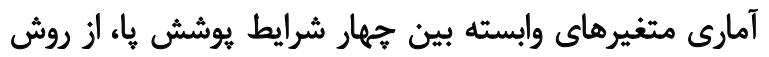

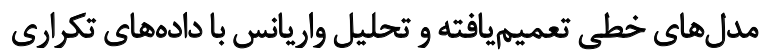

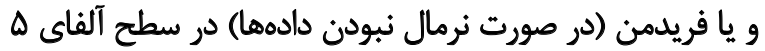

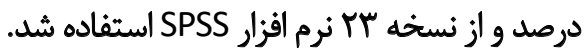

ثنايج

متغيرهاى مرتبط با فشار كف هايیى شامل مساحت سطح

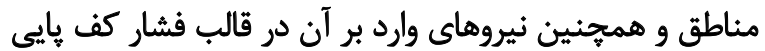
تركيب و تحليل شدند.

حداكثر فشار ناحيه شست بين سه شرايط يوشش باريا تفاوت

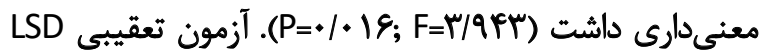
نشان داد كه ميزان حداكثر فشار كف بائى در ناحيه شست آنست

3. Extention

4. Tibial Mechanical Axis (TMA) 
جدول ا. ميانكين و انحراف استاندارد حداكثر فشار نقاط دهُائه كف باييى بين جهار شرايط مختلف حين راهرفتن

\begin{tabular}{|c|c|c|c|c|}
\hline \multicolumn{4}{|c|}{ ميانكين|نحراف اسيتاثدارد } & \multirow{2}{*}{ نيوتن بر سانتىمتر مربع } \\
\hline كفش نإيايدار توليد داخل & كفش نايايدار مشابه خارجى & كفت معمولى & ياى بوهنه & \\
\hline$\cdot / r \Delta V \pm . / \cdot r T^{* *}$ & $\Delta / \cdot \eta^{e} \pm r / M \cdot \cdots$ & D/VAq \pm T/AIr & 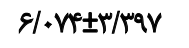 & حلاكثر فشار شست \\
\hline W/EgI士V/gF & $V / T A I \pm \cdot / r \cdot \Delta$ & E/TEN土F/T.1 & $\Delta /{ }^{\prime} U \pm \Delta / \uparrow A f^{* *}$ & حداكثر فشار انكششتان r-r \\
\hline S/OTAEM/AMr & Q/ART士 $\pm / \& N T$ & 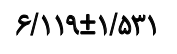 & S/ETA $1 / \cdot 19$ & حداكثر فشار مثاتارسال 1 \\
\hline VIETYEYTRAA & $\varepsilon / M^{\prime} \Delta \pm 1 / 9 \vee \varepsilon$ & 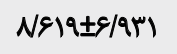 & 1./Marta/grr & حداكثر فشار متاتارسال r \\
\hline NARATr/MTA & $V / M V D \pm Y / M P I$ & $N F 8 \cdot \pm \Delta / \cdot V q$ & 1./QHIIE/MTa & حذاكثر فشار مثاثارسال r \\
\hline$r \cdot / \& E+U V / \Delta r$. & 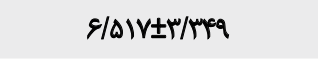 & $V / \Delta \cdot V \pm T / T Q V$ & NFT+ $\pm F l \cdot A Y$ & حداكثر فشار متأثارسال F \\
\hline NIVDEY/rVE* & g/aDr \pm V/ATr & 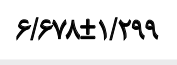 & $\Delta / \Delta \Delta+ \pm 1 / 99)^{*}$ & حداكثر فشار مثائارسال هـ \\
\hline$\Delta / T \cdot 1 \pm 1 / N \cdot 1$ & $\Delta / \cdot \cdot V \pm \cdot / V q V$ & F/rAFET/TqY & f/rTSAIV/ArA & حداكثر فشار ميانه با \\
\hline e/Rfitr/seg & $\Delta / R T I \pm T / \cdot+r$ & $\Delta / F q r \pm r / r q u$ & $s / q \subset \pm T / \varepsilon .$. & حلاكثر قشار داخل ياتشنه \\
\hline$\Delta / / A \Psi \pm Y / \Delta \backslash \Delta$ & $\Delta / \cdot \varnothing \Delta \pm T / T M$ & $\Delta / N T Y \pm T / Y+$ & S/IYEIISIR & حلاكثر فشار خارج ياشنه \\
\hline
\end{tabular}

مجله بيومكانيك ورنث

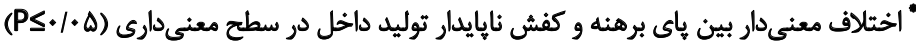

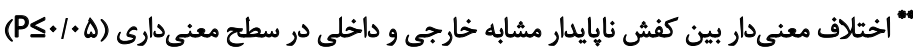

معنادارى افزايش يافت. با وجود اين، بين ساير شرايط تفاوت

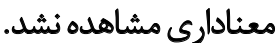

در نواحى شست يا و ناحيه انكَشتان و ناحيه متاتارسال ينجمه

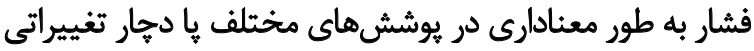

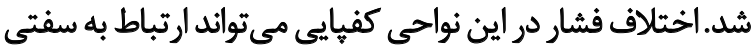

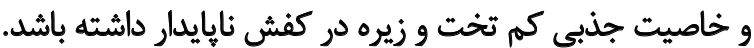

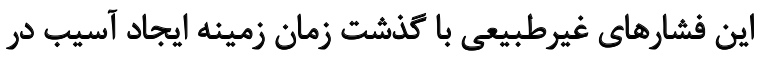

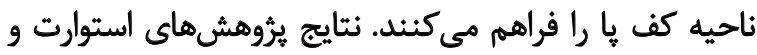

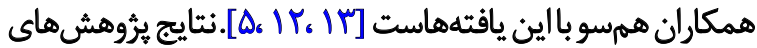

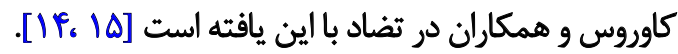
ممكن است اين اختلاف به جهت نوع ابزار استفاده براى

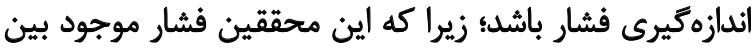

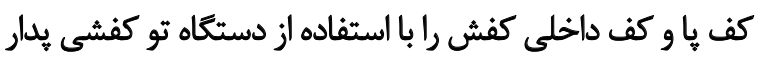

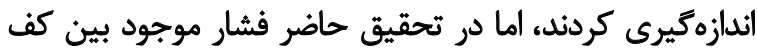

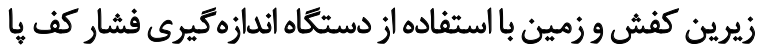
(فوت اسكن) اندازهكيرى شده است.

مغايرت برخى از نتايج تحقيق حاضر با تحقيقات ديكر نيز

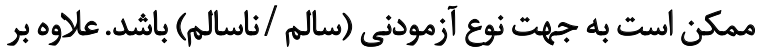

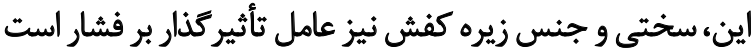

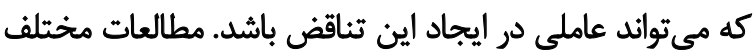

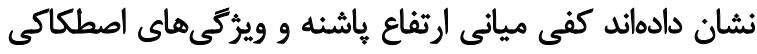

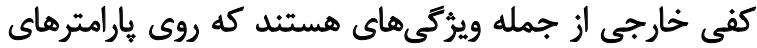

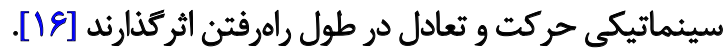

از جهت ديگر در مطالعات كذشته در كفشهاى نايايدار، كاهش
فشار ناحيه ميانى يا وجود ندارد (ه • P (PZ).

حداكثر فشار ناحيه داخلى ياشنه يا بين جهار شرايط يوشش يا

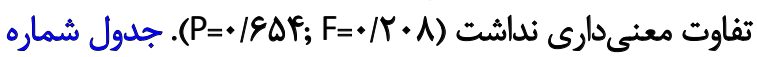

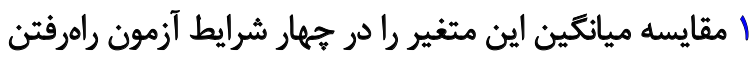

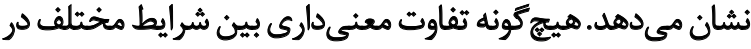

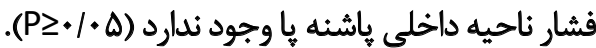

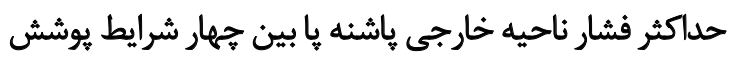

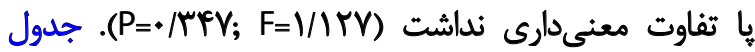

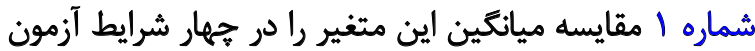

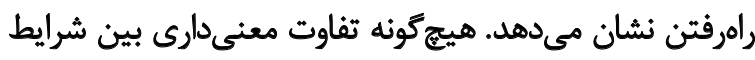

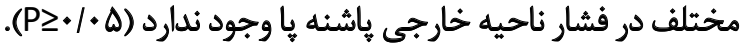

\section{بحث}

يافتههاى جدول شماره إنشان مى دهد كه حداكثر فشار كف

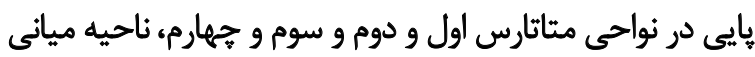

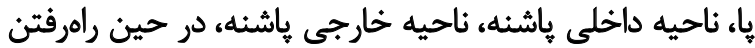

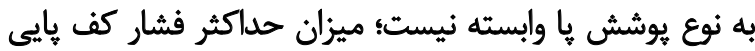

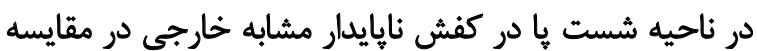

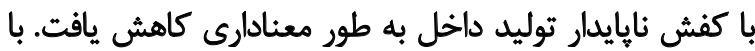

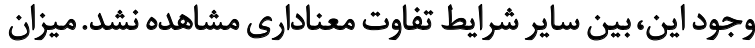

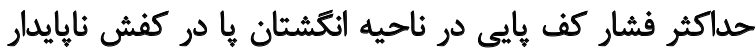

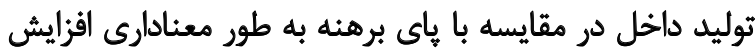

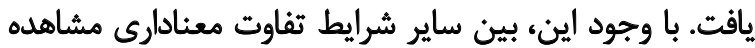

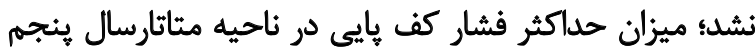

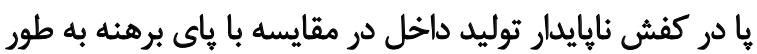




\section{نتيجه كيرى نهايی}

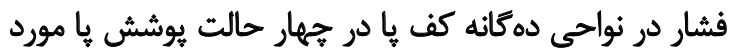

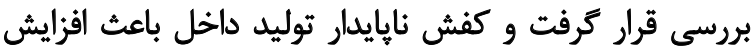

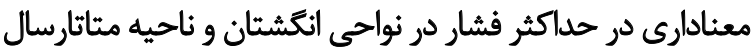

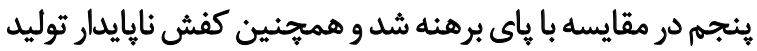

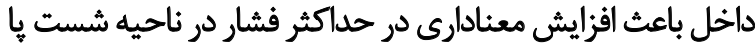

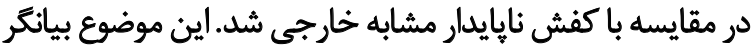

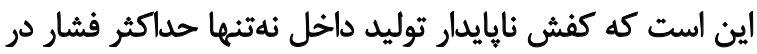

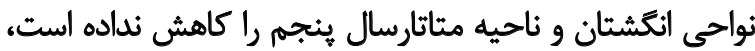

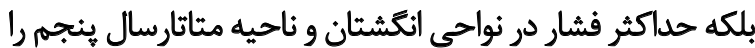

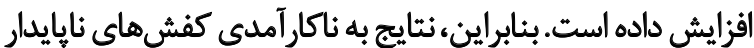
توليد داخل در تعديل فشار كفيايى در افراد اشاره بارئ دارد.

ملاحظات اخلاقى

$$
\text { يبيروى از اصول اخلاق يثوهش }
$$

رضايتنامه جهت شركت در آزمون و قبول شرايط مربوطه

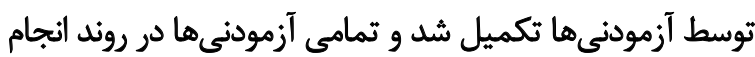
ئروهش قرار ترفتند.

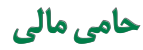

مقاله فوق بركرفته از باياننامه آقاى ساعد احمدى كنجهي

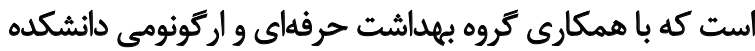

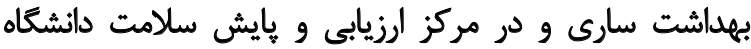

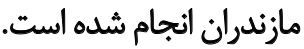

$$
\text { مشاركت نويسند }
$$

مفهومسازي: ساعد احمدى كنجه و سيد اسماعيل حسينىنرئرادي

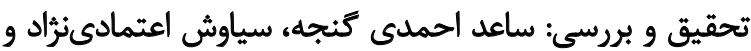

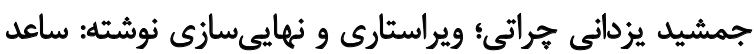
احمدى كنجه.

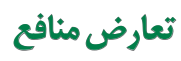

طبق نظر نويسندكان،اين مقاله هيجّونه نعارض منافعى ندارد.

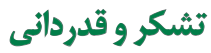

نويسندكان بر خود لازم مى دانند از همكارى مركز ارزيابى و

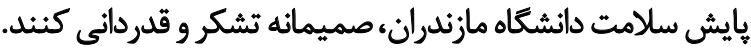

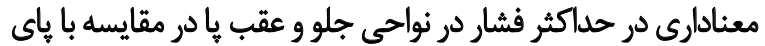

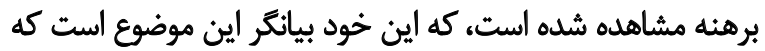

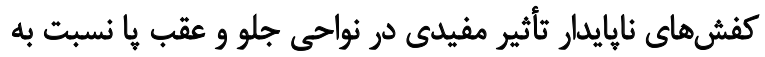

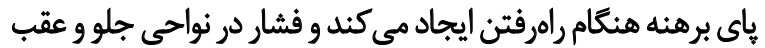

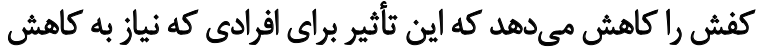

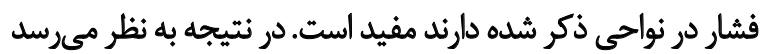

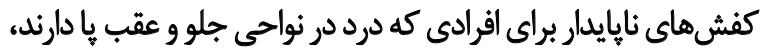

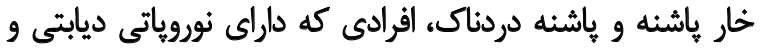

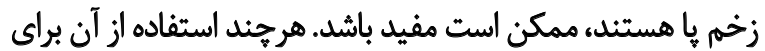
مشكلات ناحيه ميانى توصيه نمى مشود.

نيروهاى برخورد به عنوان يكى از مهمرترين عوامل ايجاد و

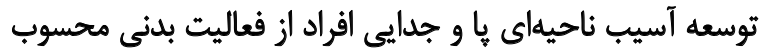

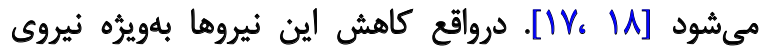

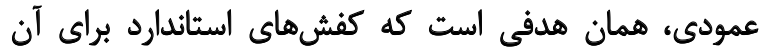

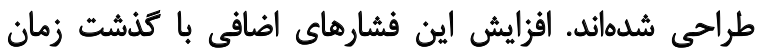

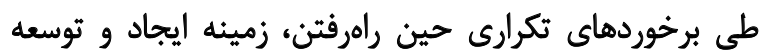

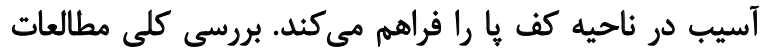

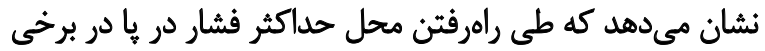

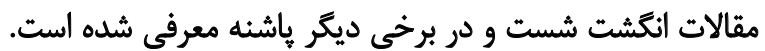

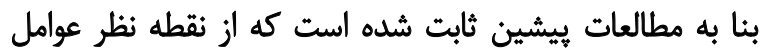

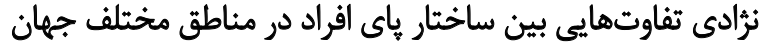

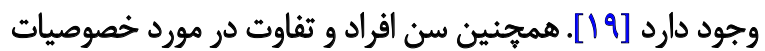

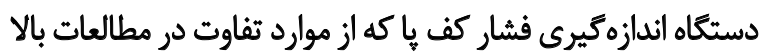

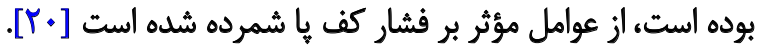
در ميان انكشتان،انتشت شست نسبت به سايرين، تحمل وزن

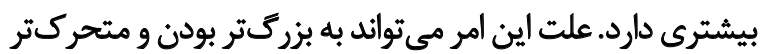

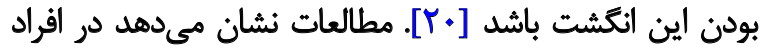

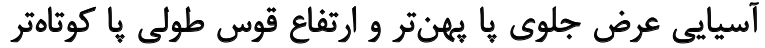

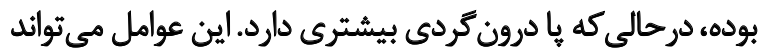

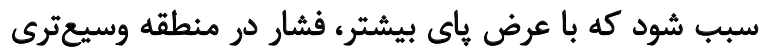

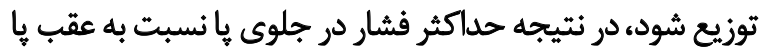

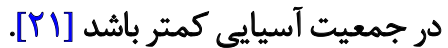

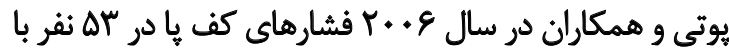

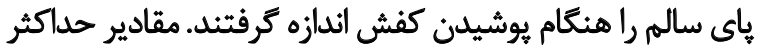

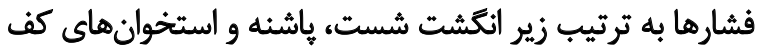

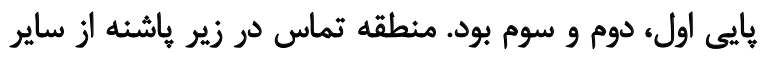

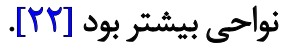

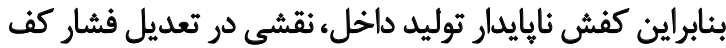

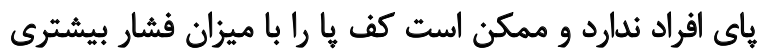

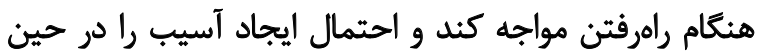

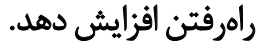




\section{References}

[1] Christopher LV, Brian L, Jeremy C. Dynamics of human gait. Cape Town, South Africa: Kiboho Publishers; 1999.

[2] Chapman J, Preece S, Braunstein B, Höhne A, Nester C, Brueggemann $P$, et al. Effect of rocker shoe design features on forefoot plantar pressures in people with and without diabetes. Clinical Biomechanics. 2013; 28(6):679-85. [DOI:10.1016/j.clinbiomech.2013.05.005] [PMID]

[3] Chen YC, Lou SZ, Huang CY, Su FC. Effects of foot orthoses on gait patterns of flat feet patients. Clinical Biomechanics. 2010; 25(3):265-70. [DOI:10.1016/j.clinbiomech.2009.11.007] [PMID]

[4] Hennig EM, Valiant GA, Liu Q. Biomechanical variables and the perception of cushioning for running in various types of footwear. Journal of Applied Biomechanics. 1996; 12(2):143-50. [DOI:10.1123/jab.12.2.143]

[5] Che H, Nigg B, De Koning J. Relationship between plantar pressure distribution under the foot and insole comfort. Clinical Biomechanics. 1994; 9(6):335-41. [DOI:10.1016/02680033(94)90062-0]

[6] Nigg BM, Emery C, Hiemstra LA. Unstable shoe construction and reduction of pain in osteoarthritis patients. Medicine \& Science in Sports \& Exercise. 2006; 38(10):1701-8. [DOI:10.1249/01.mss.0000228364.93703.53] [PMID]

[7] Stewart L, Gibson J, Thomson CE. In-shoe pressure distribution in "unstable" (MBT) shoes and flat-bottomed training shoes: A comparative study. Gait \& Posture. 2007; 25(4):648-51. [DOI:10.1016/j.gaitpost.2006.06.012] [PMID]

[8] Romkes J, Rudmann C, Brunner R. Changes in gait and EMG when walking with the Masai Barefoot Technique. Clinical Biomechanics. 2006; 21(1):75-81. [DOI:10.1016/j.clinbiomech.2005.08.003] [PMID]

[9] Eslami M, Hoseini Nejad SE, Gandomkar A, Jahedi V. [Effect of unstable shoes on ground reaction force parameters during stance phase of running (Persian)]. Journal Researches in Sport Medicine and Technology. 2013; 11(6):90-101.

[10] Gandomkar A, Eslami M, Hoseini Nejad SE, Jahedi V. [Effect of unstable shoes on lower extremity joint power during stance phase of running (Persian)]. Razi Journal of Medical Sciences. 2014; 21(124):54-63.

[11] Barrios JA, Heitkamp CA, Smith BP, Sturgeon MM, Suckow DW, Sutton CR. Three-dimensional hip and knee kinematics during walking, running, and single-limb drop landing in females with and without genu valgum. Clinical Biomechanics. 2016; 31:7-11. [DOI:10.1016/j.clinbiomech.2015.10.008] [PMID]

[12] An S, Lee K. Effect of rocker heel angle of walking shoe on gait mechanics and muscle activity. In: Fuss FK, Subic A, Ujihashi S, editors. The Impact of Technology on Sport II. London: Taylor \& Francis; 2007

[13] Stöggl T, Haudum A, Birklbauer J, Murrer M, Müller E. Short and long term adaptation of variability during walking using unstable (Mbt) shoes. Clinical Biomechanics. 2010; 25(8):816-22. [DOI:10.1016/j.clinbiomech.2010.05.012] [PMID]
[14] Nigg B, Hintzen S, Ferber R. Effect of an unstable shoe construction on lower extremity gait characteristics. Clinical Biomechanics. 2006; 21(1):82-8. [DOI:10.1016/j.clinbiomech.2005.08.013] [PMID]

[15] Kavros SJ, Van Straaten MG, Wood KAC, Kaufman KR. Forefoot plantar pressure reduction of off-the-shelf rocker bottom provisional footwear. Clinical Biomechanics. 2011; 26(7):778-82. [DOI:10.1016/j.clinbiomech.2011.03.009] [PMID]

[16] Perry SD, Radtke A, Goodwin CR. Influence of footwear midsole material hardness on dynamic balance control during unexpected gait termination. Gait \& Posture. 2007; 25(1):94-8. [DOI:10.1016/j.gaitpost.2006.01.005] [PMID]

[17] Nigg BM. Biomechanics of sport shoes. Calgary: Topline Printing Inc; 2010.

[18] Chuckpaiwong B, Cook C, Pietrobon R, Nunley JA. Second metatarsal stress fracture in sports: Comparative risk factors between proximal and non-proximal locations. British Journal of Sports Medicine. 2007; 41(8):510-4. [DOI:10.1136/ bjsm.2006.033571] [PMID] [PMCID]

[19] Logan S, Hunter I, Hopkins JT, Feland JB, Parcell AC. Ground reaction force differences between running shoes, racing flats, and distance spikes in runners. Journal of sports science \& medicine. 2010; 9(1):147-53. [PMCID] [PMID]

[20] Hennig EM. The human foot during locomotion-applied research for footwear. Hong Kong: The Chinese University of Hong Kong; 2002.

[21] Perttunen J. Foot loading in normal and pathological walking [PhD. dissertation]. Jyväskylä: University of Jyväskylä; 2002.

[22] Putti A, Arnold G, Cochrane L, Abboud R. The Pedar ${ }^{\circledR}$ in-shoe system: Repeatability and normal pressure values. Gait \& Posture. 2007; 25(3):401-5. [DOI:10.1016/j.gaitpost.2006.05.010] [PMID] 
This Page Intentionally Left Blank 